\title{
Approaching the $N=82$ shell closure with mass measurements of Ag and Cd isotopes
}

\author{
M. Breitenfeldt, ${ }^{1, *}$ Ch. Borgmann, ${ }^{2}$ G. Audi, ${ }^{3}$ S. Baruah,${ }^{1, \dagger}$ D. Beck, ${ }^{4}$ K. Blaum,${ }^{2,5}$ Ch. Böhm, ${ }^{2}$ R. B. Cakirli,,${ }^{2,6}$ \\ R. F. Casten, ${ }^{7}$ P. Delahaye, ${ }^{8, \ddagger}$ M. Dworschak, ${ }^{4}$ S. George, ${ }^{2,}{ }^{\circledR}$ F. Herfurth, ${ }^{4}$ A. Herlert ${ }^{8}$ A. Kellerbauer, ${ }^{9}$ M. Kowalska, ${ }^{8}$ \\ D. Lunney, ${ }^{3}$ E. Minaya-Ramirez, ${ }^{3}$ S. Naimi, ${ }^{3}$ D. Neidherr, ${ }^{10}$ M. Rosenbusch, ${ }^{1}$ R. Savreux, ${ }^{4}$ S. Schwarz, ${ }^{11}$ \\ L. Schweikhard, ${ }^{1}$ and C. Yazidjian ${ }^{4}$ \\ ${ }^{1}$ Institut für Physik, Ernst-Moritz-Arndt-Universität, D-17487 Greifswald, Germany \\ ${ }^{2}$ Max-Planck-Institut für Kernphysik, Saupfercheckweg 1, D-69117 Heidelberg, Germany \\ ${ }^{3}$ CSNSM-IN2P3-CNRS, Université de Paris Sud, F-91405 Orsay, France \\ ${ }^{4}$ GSI Helmholtzzentrum für Schwerionenforschung GmbH, Planckstraße 1, D-64291 Darmstadt, Germany \\ ${ }^{5}$ Ruprecht-Karls-Universität, Physikalisches Institut, D-69120 Heidelberg, Germany \\ ${ }^{6}$ Department of Physics, University of Istanbul, Istanbul, Turkey \\ ${ }^{7}$ Wright Nuclear Structure Laboratory, Yale University, New Haven, Connecticut 06520, USA \\ ${ }^{8}$ CERN, Physics Department, 1211 Geneva 23, Switzerland \\ ${ }^{9}$ Commission European Communities, Joint Research Center, European Institute for Transuranium Elements, D-76125 Karlsruhe, Germany \\ ${ }^{10}$ Institut für Physik, Johannes Gutenberg-Universität, D-55128 Mainz, Germany \\ ${ }^{11}$ NSCL, Department of Physics and Astronomy, Michigan State University, Michigan 48824, East Lansing, USA
}

(Received 13 November 2009; published 26 March 2010)

\begin{abstract}
Mass measurements of neutron-rich $\mathrm{Cd}$ and $\mathrm{Ag}$ isotopes were performed with the Penning trap mass spectrometer ISOLTRAP. The masses of ${ }^{112,114-124} \mathrm{Ag}$ and ${ }^{114,120,122-124,126,128} \mathrm{Cd}$, determined with relative uncertainties between $2 \times 10^{-8}$ and $2 \times 10^{-7}$, resulted in significant corrections and improvements of the mass surface. In particular, the mass of ${ }^{124} \mathrm{Ag}$ was previously unknown. In addition, other masses that had to be inferred from $Q$ values of nuclear decays and reactions have now been measured directly. The analysis includes various mass differences, namely the two-neutron separation energies, the applicability of the Garvey-Kelson relations, double differences of masses $\delta V_{p n}$, which give empirical proton-neutron interaction strengths, as well as a comparison with recent microscopic calculations. The $\delta V_{p n}$ results reveal that for even-even nuclides around ${ }^{132} \mathrm{Sn}$ the trends are similar to those in the ${ }^{208} \mathrm{~Pb}$ region.
\end{abstract}

DOI: 10.1103/PhysRevC.81.034313

PACS number(s): 21.10.Dr, 21.30.Fe, 27.60.+j

\section{INTRODUCTION}

Direct mass measurements allow an accurate determination of the nuclear binding energy. Present experimental studies reach an accuracy of a few $\mathrm{eV}$ for stable nuclides [1-3] and about $100 \mathrm{eV}$ for radionuclides [4-8]. Mass values of such precision allow nuclear structure studies. For example, mass values determined at ISOLTRAP [9] provided important information concerning the question of shell closures [10].

A mass filter $\delta V_{p n}$, obtained from double differences of binding energies, can be constructed that isolates the interactions of the last protons and neutrons [11]. Recent surveys [12,13] using the Atomic-Mass Evaluation 2003 (AME2003) [14] yielded interesting results concerning the influences of the orbits of the valence nucleons on the protonneutron $(\mathrm{p}-\mathrm{n})$ interaction strengths in the ${ }^{208} \mathrm{~Pb}$ mass region,

\footnotetext{
*Corresponding author: Martin.Breitenfeldt@cern.ch; present address: Instituut voor Kern- en Stralingsfysica, Katholieke Universiteit Leuven, Celestijnenlaan 200D, B-3001 Leuven, Belgium.

†Present address: Bhabha Atomic Research Centre, Trombay, 400085 Mumbai, India.

${ }^{\ddagger}$ Present address: Grand Accélérateur National d'Ions Lourds, 14076 CAEN Cedex 05, France.

§Present address: NSCL, Department of Physics and Astronomy, Michigan State University, MI 48824, East Lansing, USA.
}

leading to characteristic patterns when magic neutron and proton numbers were approached and crossed. Furthermore, the application of $\delta V_{p n}$ in the vicinity of the neutron-rich $\mathrm{Rn}$ nuclides suggested this interaction filter may be sensitive to octupole correlations [15]. Finally, these p-n interactions can be confronted by microscopic calculations using various interactions.

A more detailed investigation by new mass measurements was suggested by Brenner et al. [13] to further investigate these trends. Some of those masses have since been measured, for example, ${ }^{208} \mathrm{Hg}$ [16] using the experimental storage ring (ESR) facility at GSI [17]. The suggestions of further mass measurements included the nuclides ${ }^{122,124,126,128} \mathrm{Cd}$ [13] for testing the behavior of $\delta V_{p n}$ in the vicinity of the Sn nuclides and for comparing those to $\delta V_{p n}$ in the $\mathrm{Pb}$ region. For the above-mentioned $\mathrm{Cd}$ nuclides we report new mass values, as well as for neutron-rich $\mathrm{Ag}$ isotopes. In the case of ${ }^{124} \mathrm{Ag}$ the mass was determined for the first time.

\section{ISOLTRAP EXPERIMENT}

The experiments were performed at the triple-trap mass spectrometer ISOLTRAP [9]. The setup is installed at the online isotope separator ISOLDE [18], which is devoted to the production of radioactive ions at CERN (Geneva). The short-lived nuclides were created by a $1.4-\mathrm{GeV}$ proton 
TABLE I. Information on the six online experiments, the investigated radionuclides, the applied target/ion source combinations, and the separator used. All experiments employed the neutron converter (n-conv.) [31] for the production of neutron-rich fission products and in the case of all Cd measurements, a temperature-controlled quartz transfer-line (QTL) was used [21]. Ionization was performed using the RILIS [19,20].

\begin{tabular}{|c|c|c|c|c|c|}
\hline Experiment \# & Date & Nuclides & Target & Ion source & Separator \\
\hline 1 & $10 / 2005$ & ${ }^{114,120,122,123} \mathrm{Cd}$ & $\mathrm{UC}_{x} \& \mathrm{n}$-conv. \& QTL & RILIS & HRS \\
\hline 2 & $05 / 2006$ & ${ }^{112,114,116,118,120,121} \mathrm{Ag}$ & $\mathrm{UC}_{x} \& \mathrm{n}$-conv. & RILIS & HRS \\
\hline 3 & $07 / 2006$ & ${ }^{124,126} \mathrm{Cd}$ & $\mathrm{UC}_{x} \& \mathrm{n}$-conv. \& QTL & RILIS & HRS \\
\hline 4 & $05 / 2007$ & $115,117,119-121,123 \mathrm{Ag}$ & $\mathrm{UC}_{x} \& \mathrm{n}$-conv. & RILIS & HRS \\
\hline 5 & $05 / 2008$ & ${ }^{126,128} \mathrm{Cd}$ & $\mathrm{UC}_{x} \& \mathrm{n}$-conv. \& QTL & RILIS & HRS \\
\hline 6 & $06 / 2009$ & ${ }^{122-124} \mathrm{Ag}$ & $\mathrm{UC}_{x} \& \mathrm{n}$-conv. & RILIS & GPS \\
\hline
\end{tabular}

beam impinging on a thick uranium-carbide target and were subsequently ionized by use of a resonance-ionization laser ion source (RILIS) [19,20], where, with up to three different laser wavelengths, the atoms are element-selectively excited into auto-ionizing states. However, a background of surfaceionized Cs contamination is also created due to the heated transfer line between the target and ion-source cavity. For the experiments on $\mathrm{Cd}$ a temperature-controlled quartz transfer line (QTL) was implemented to reduce the Cs background by a factor of $\approx 600$ [21]. Table I shows the investigated nuclides and the target/ion source combinations used in the experiments of the present work.

The ions were accelerated to an energy between 30 and $50 \mathrm{keV}$ mass separated and transported to the ISOLTRAP setup via the High Resolution Separator (HRS) or the General Purpose Separator (GPS), which have mass resolving powers of $m / \Delta m \simeq 3000$ and 1000 , respectively.

ISOLTRAP consists of three main functional parts (Fig. 1). In the first stage, a radio-frequency quadrupole cooler and buncher (RFQ buncher) [22] operated at a potential between 30 and $50 \mathrm{kV}$, the ion beam is stopped and converted into an ion bunch with a small radial and axial emittance [23]. The ion bunches are transported at low energy to a cylindrical preparation Penning trap [24], where a massselective buffer-gas cooling technique [25] is applied to rid the bunch of isobaric contamination. A resolving power of about 40,000 was used for the present measurements. For the mass determination in the hyperbolic measurement Penning trap, the cyclotron frequency $v_{c}=q B /(2 \pi m)$ of the ions is probed by the use of the time-of-flight ion-cyclotron-resonance (ToF-ICR) technique. The ion motion is manipulated by applying a radial quadrupolar radio frequency (rf) voltage. The ions are ejected from the Penning trap and their ToF is recorded. The resonantly excited ions $\left(v_{r f}=v_{c}\right)$ have the shortest ToF to the detector [26], a channeltron with nearly $100 \%$ detection efficiency [27] (except for the experiment in June 2009, where a multichannel plate detector was used). The dependence of the ToF on the excitation frequency is well known [28] and a fit of the resonance curve to the measured ToF values leads to a precise determination of the cyclotron frequency $\nu_{c}$. In the inset of Fig. 1 ToF-ICR data for ${ }^{128} \mathrm{Cd}^{+}$ and the fit curve are plotted. For a mass determination the magnetic field strength is interpolated between measurements of the reference ion ${ }^{133} \mathrm{Cs}^{+}$before and after each resonance of the ions of interest. The absence of resonant-frequency shifts in all the data indicates that no contamination was present [29]. To account for unknown systematic effects a mass-independent systematic uncertainty of $8 \times 10^{-9}$ [29] is added. (This point is discussed in more detail in Ref. [30].)

\section{RESULTS: MASS VALUES AND ATOMIC-MASS EVALUATION}

The experimental results were obtained during six different online experiments between October 2005 and June 2009 (see Table I). In general, at least two resonances were taken for each of the investigated $\mathrm{Ag}$ and $\mathrm{Cd}$ nuclides (except in
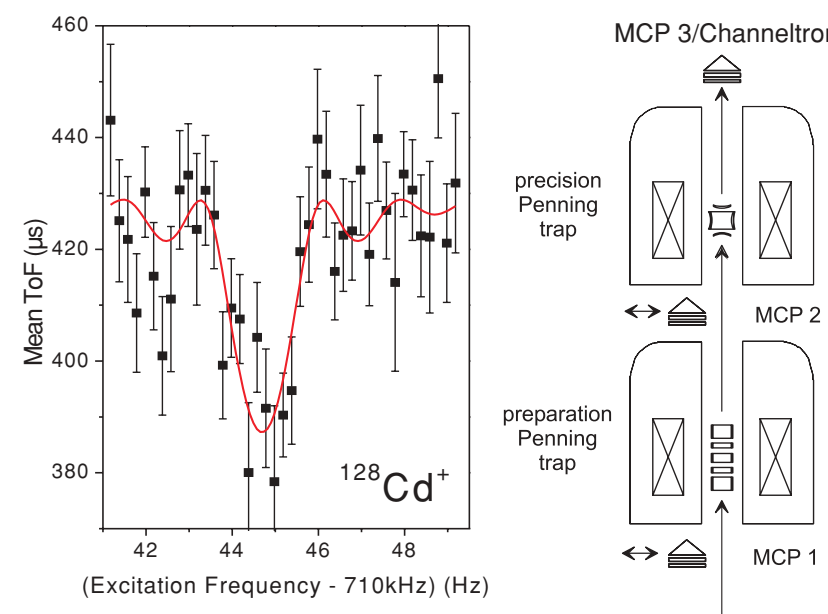

(Excitation Frequency $-710 \mathrm{kHz})(\mathrm{Hz})$ 2nd pulsed

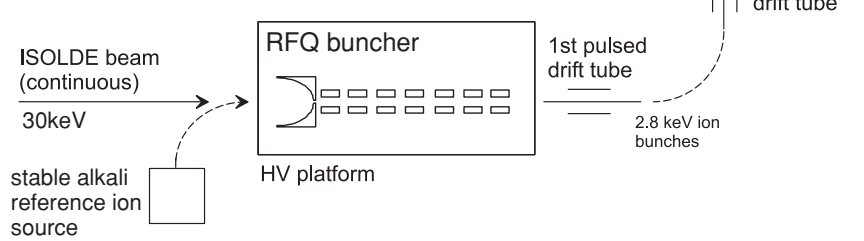
source

FIG. 1. (Color online) The triple-trap mass spectrometer ISOLTRAP consisting of an RFQ buncher and two Penning traps. The inset shows a recorded time-of-flight ion-cyclotron resonance for ${ }^{128} \mathrm{Cd}^{+}$fit with the theoretical lineshape (solid line) [28] to the data. The quadrupolar rf excitation was performed for $600 \mathrm{~ms}$. 
TABLE II. Half-lives and ratios $r=v_{c}\left({ }^{133} \mathrm{Cs}^{+}\right) / v_{c}\left({ }^{\mathrm{A}}\{\mathrm{Ag}, \mathrm{Cd}\}^{+}\right)$ between the cyclotron frequencies of the reference nuclide ${ }^{133} \mathrm{Cs}$ and the neutron-rich $\mathrm{Ag}$ and $\mathrm{Cd}$ nuclides.

\begin{tabular}{lccc}
\hline \hline Nuclide & Experiment \# & Half-life & \multicolumn{1}{c}{$r$} \\
\hline${ }^{112} \mathrm{Ag}$ & 2 & $3.130(9) \mathrm{h}$ & $0.8420042984(193)$ \\
${ }^{114} \mathrm{Ag}$ & 2 & $4.6(1) \mathrm{s}$ & $0.8570660039(369)$ \\
${ }^{115} \mathrm{Ag}$ & 4 & $53.46(10) \mathrm{h}$ & $0.8645900022(692)$ \\
${ }^{116} \mathrm{Ag}$ & 2 & $2.68(19) \mathrm{min}$ & $0.8721336481(261)$ \\
${ }^{117} \mathrm{Ag}$ & 4 & $73.6(1.4) \mathrm{s}$ & $0.8796608164(298)$ \\
${ }^{118} \mathrm{Ag}$ & 2 & $3.76(15) \mathrm{s}$ & $0.8872071748(200)$ \\
${ }^{119} \mathrm{Ag}$ & 4 & $6.0(5) \mathrm{s}$ & $0.8947377132(430)$ \\
${ }^{120} \mathrm{Ag}$ & 2 & $1.23(4) \mathrm{s}$ & $0.9022860189(363)$ \\
${ }^{120} \mathrm{Ag}$ & 4 & $1.23(4) \mathrm{s}$ & $0.9022861644(922)$ \\
${ }^{121} \mathrm{Ag}$ & 2 & $790(20) \mathrm{ms}$ & $0.9098203654(378)$ \\
${ }^{121} \mathrm{Ag}$ & 4 & $790(20) \mathrm{ms}$ & $0.9098204035(896)$ \\
${ }^{122} \mathrm{Ag}$ & 6 & $520(14) \mathrm{ms}$ & $0.917371415(138)$ \\
${ }^{123} \mathrm{Ag}$ & 4 & $296(6) \mathrm{ms}$ & $0.924907151(867)$ \\
${ }^{123} \mathrm{Ag}$ & 6 & $296(6) \mathrm{ms}$ & $0.924907931(228)$ \\
${ }^{124} \mathrm{Ag}$ & 6 & $172(5) \mathrm{ms}$ & $0.93245906(201)$ \\
${ }^{114} \mathrm{Cd}$ & 1 & stable & $0.8570249064(316)$ \\
${ }^{120} \mathrm{Cd}$ & 1 & $50.80(21) \mathrm{s}$ & $0.9022189281(304)$ \\
${ }^{122} \mathrm{Cd}$ & 1 & $5.24(3) \mathrm{s}$ & $0.9172942676(351)$ \\
${ }^{123} \mathrm{Cd}$ & 1 & $2.10(2) \mathrm{s}$ & $0.924845957(198)$ \\
${ }^{124} \mathrm{Cd}$ & 3 & $1.25(2) \mathrm{s}$ & $0.9323742835(766)$ \\
${ }^{126} \mathrm{Cd}$ & 1 & $517(17) \mathrm{ms}$ & $0.9474585036(340)$ \\
${ }^{126} \mathrm{Cd}$ & 5 & $517(17) \mathrm{ms}$ & $0.947458423(116)$ \\
${ }^{128} \mathrm{Cd}$ & 5 & $280(40) \mathrm{ms}$ & $0.9625472948(818)$ \\
\hline \hline & & &
\end{tabular}

the case of ${ }^{115} \mathrm{Ag}$, where only a single measurement was performed). The averaged values of the frequency ratios $r=v_{c}\left({ }^{133} \mathrm{Cs}^{+}\right) / v_{c}\left({ }^{\mathrm{A}}\{\mathrm{Ag}, \mathrm{Cd}\}^{+}\right)$between the cyclotron frequencies of the reference nuclide ${ }^{133} \mathrm{Cs}$ and the neutron-rich $\mathrm{Ag}$ and $\mathrm{Cd}$ nuclides are given in Table II along with the half-lives.

Because of possibly unresolved isomeric states, the experimentally determined mass-excess values of ${ }^{115,117} \mathrm{Ag}$ and ${ }^{123} \mathrm{Cd}$ were treated as described by Wapstra et al. [32]. The mass-excess values were increased by half of the excitation energy of the potentially present excited isomeric state. For the correction of the uncertainties of the experimental data, two cases were considered: First, a pure ion ensemble was present, but no information for an assignment of the measured value to a state was available. In this case the mass uncertainty was increased by quadratically adding the uncertainty of the excitation energy and half of the excitation energy of the isomer. Second, the ion ensemble is a mixture of ions in the ground state and in the excited state. In this case the experimental uncertainty was increased by 0.29 of the excitation energy. In Table III nuclides of the first case are labeled ${ }^{x}$ and the second case by ${ }^{y}$, whereas the ground state is indicated by ${ }^{g}$ and the first excited state by ${ }^{m}$.

For the cases of ${ }^{119,121-124} \mathrm{Ag}$ isomeric states were not yet observed, but following the systematic behavior shown in Fig. 2 for even- $N$ Ag nuclides, we assumed that such isomers exist. In this figure the difference between the level energies of the lowest-lying $1 / 2^{-}$and $7 / 2^{+}$states is plotted, which are the ground and the first excited states. From this trend

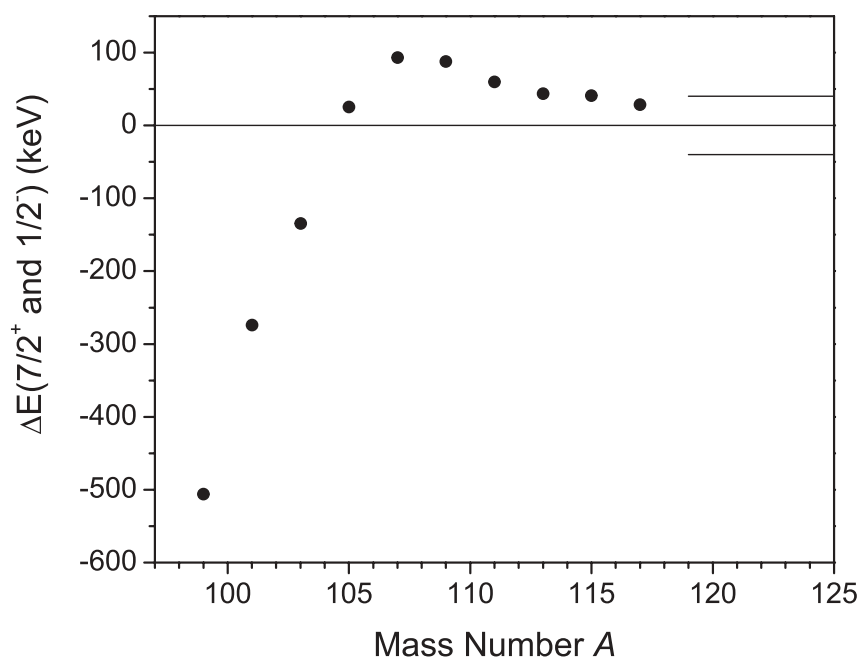

FIG. 2. Energy separation between the lowest-lying $7 / 2^{+}$and $1 / 2^{-}$states of even- $N \mathrm{Ag}$ isomers as a function of the atomic mass number. The negative energies for the first three values result from an inversion of the spins of the ground state and first excited state from ${ }^{103} \mathrm{Ag}$ to ${ }^{105} \mathrm{Ag}$. The short horizontal lines for neutron-rich nuclides with $A>118$ show the assumed range of excitation energies for the isomers.

the excitation energies of the isomeric states in ${ }^{121} \mathrm{Ag}$ and ${ }^{123} \mathrm{Ag}$ are estimated conservatively with an excitation energy of about 20(20) $\mathrm{keV}$. This procedure was already applied for $119,122,124 \mathrm{Ag}$ in the AME2003 [14].

For ${ }^{118} \mathrm{Ag}$ the measured value was assigned to the isomeric state due to the agreement with the known mass-excess value [14]. In addition, the value of the excitation energy of $\approx 128 \mathrm{keV}$ is more than an order of magnitude higher than the uncertainty of the measurement. Finally, with the standard analysis procedure [29] no hint of close-lying contamination was found.

The measured frequency ratios from this work were included into a new atomic-mass evaluation, which was performed as in previous treatments (e.g., in Refs. [33,34]). This procedure gives the currently best mass values. The result also shows the influence of the new data on the mass network. In Fig. 3 and Table III the new mass-excess values are compared with those derived from the ISOLTRAP data and with the values of the AME2003 [14]. In the following, all investigated nuclides are discussed in turn. The influences of the contributing data to the final mass-excess value, which results from the flow-of-information matrix [35], is given as a percentage. In general these values are rounded and thus their sum is not always exactly $100 \%$.

\section{A. The Ag isotopes}

\section{${ }^{112} \mathrm{Ag}$}

The AME2003 mass-excess value and the ISOLTRAP value deviate by about $40 \mathrm{keV}(2.3 \sigma)$. In the AME2003 the massexcess value was determined with a weighting of $69.7 \%$ by the $Q$ value [299(20) $\mathrm{keV}]$ of the $\beta$ decay ${ }^{112} \mathrm{Ag}\left(\beta^{-}\right){ }^{112} \mathrm{Cd}[36]$ 
TABLE III. Mass excesses $M E$ of neutron-rich $\mathrm{Cd}$ and $\mathrm{Ag}$ isotopes as determined by ISOLTRAP (not corrected for isomeric mixtures) and of AME2003, the excitation energies of the isomeric states, the $M E$ value determined by ISOLTRAP but corrected for the possible presence of isomers, and a new atomic-mass evaluation. Nuclides where the isomeric state cannot be assigned are referred to with ${ }^{x}$ and isomeric admixtures with ${ }^{y}$, whereas the ground state is indicated with ${ }^{g}$ and the first excited state is indicated with ${ }^{m}$.

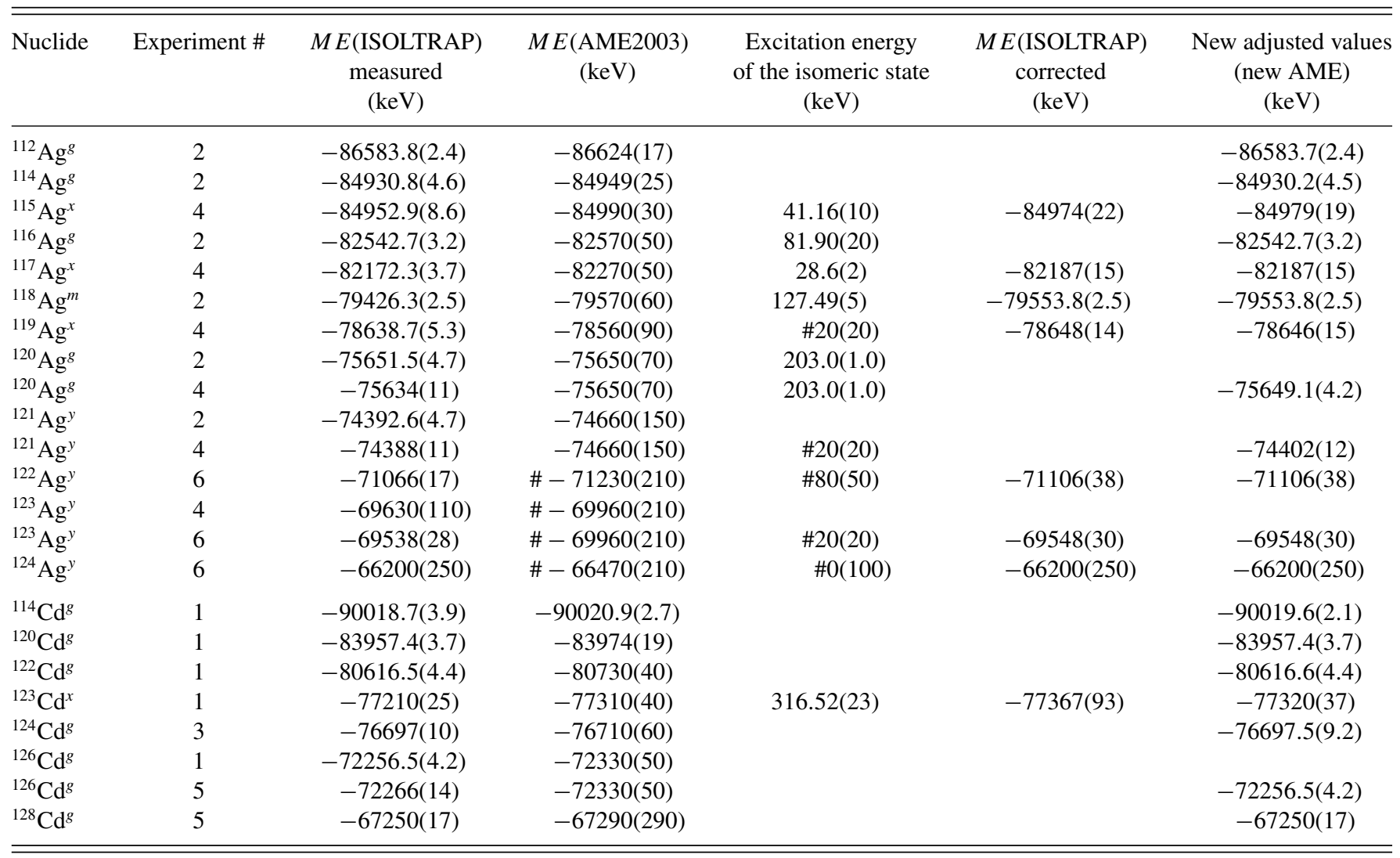

and with a weighting of $30.3 \%$ by the $Q$ value [3967(20) $\mathrm{keV}$ ] of the decay ${ }^{112} \mathrm{Pd}\left(\beta^{-}\right)^{112} \mathrm{Ag}[37]$. In the new evaluation the mass is fully determined by the ISOLTRAP value.

${ }^{114} \mathrm{Ag}$

The mass-excess value from ISOLTRAP and the one from AME2003 agree within their uncertainties. The AME2003 mass was determined with a weighting of $50.3 \%$ from the $\beta^{-}$decay of ${ }^{114} \mathrm{Pd}[38,39]$ with $Q$ values of $1414(30)$ and $1451(25) \mathrm{keV}$, respectively, and with a weighting of $49.7 \%$ by the $\beta^{-}$decay of ${ }^{114} \mathrm{Ag}$ [38] with a $Q$ value of $5018(35) \mathrm{keV}$. After the new evaluation the ${ }^{114} \mathrm{Ag}$ mass is almost entirely determined by the ISOLTRAP value (98.3\%). A contribution of $1.7 \%$ from ${ }^{114} \mathrm{Pd}\left(\beta^{-}\right)^{114} \mathrm{Ag}$ remains. This connection contributes to the mass-excess value of ${ }^{114} \mathrm{Pd}$, which is now $-83483(16) \mathrm{keV}$ as compared to the AME2003 value of $-83497(24) \mathrm{keV}$.

$$
{ }^{115} \mathrm{Ag}
$$

The ISOLTRAP value was corrected to account for a possible mixture of the ground state and the isomeric state with an excitation energy of 41.16(10) $\mathrm{keV}$. The corrected value agrees with the value from AME2003, which was determined by three studies of the decay ${ }^{115} \mathrm{Ag}\left(\beta^{-}\right){ }^{115} \mathrm{Cd}$ $[38,40,41]$ with the $Q$ values of $3180(100), 3105(100)$, and
3091(40) keV, respectively. The corrected ISOLTRAP value contributes $76.3 \%$ to the value of the new AME whereas the remaining $23.8 \%$ comes from the $\beta$-decay $Q$ values.

\section{${ }^{116} \mathrm{Ag}$}

The ISOLTRAP mass excess agrees with the AME2003 value, determined by two $Q$ values of the $\beta^{-}$decay of ${ }^{116} \mathrm{Ag}$ $[38,42][6028(130)$ and 6170(50) $\mathrm{keV}]$. The value of the new AME is entirely determined by ISOLTRAP.

\section{${ }^{117} \mathrm{Ag}$}

Due to the presence of a long-lived isomeric state ${ }^{117} \mathrm{Ag}^{m}$ with an excitation energy of 28.6(0.2) keV the ISOLTRAP mass-excess value was corrected. This value agrees within the uncertainties with the value of the AME2003, determined completely by the ${ }^{117} \mathrm{Ag}\left(\beta^{-}\right){ }^{117} \mathrm{Cd} Q$ value of $4160(50) \mathrm{keV}$ [42]. The value of the new AME is entirely determined by the corrected ISOLTRAP value.

\section{${ }^{118} \mathrm{Ag}$}

The mass-excess value determined with ISOLTRAP was assigned to the isomeric state with an excitation energy of $127.49(0.05) \mathrm{keV}$. The resulting mass-excess value agrees with the AME2003 value, determined by the link to ${ }^{118} \mathrm{Cd}$ 

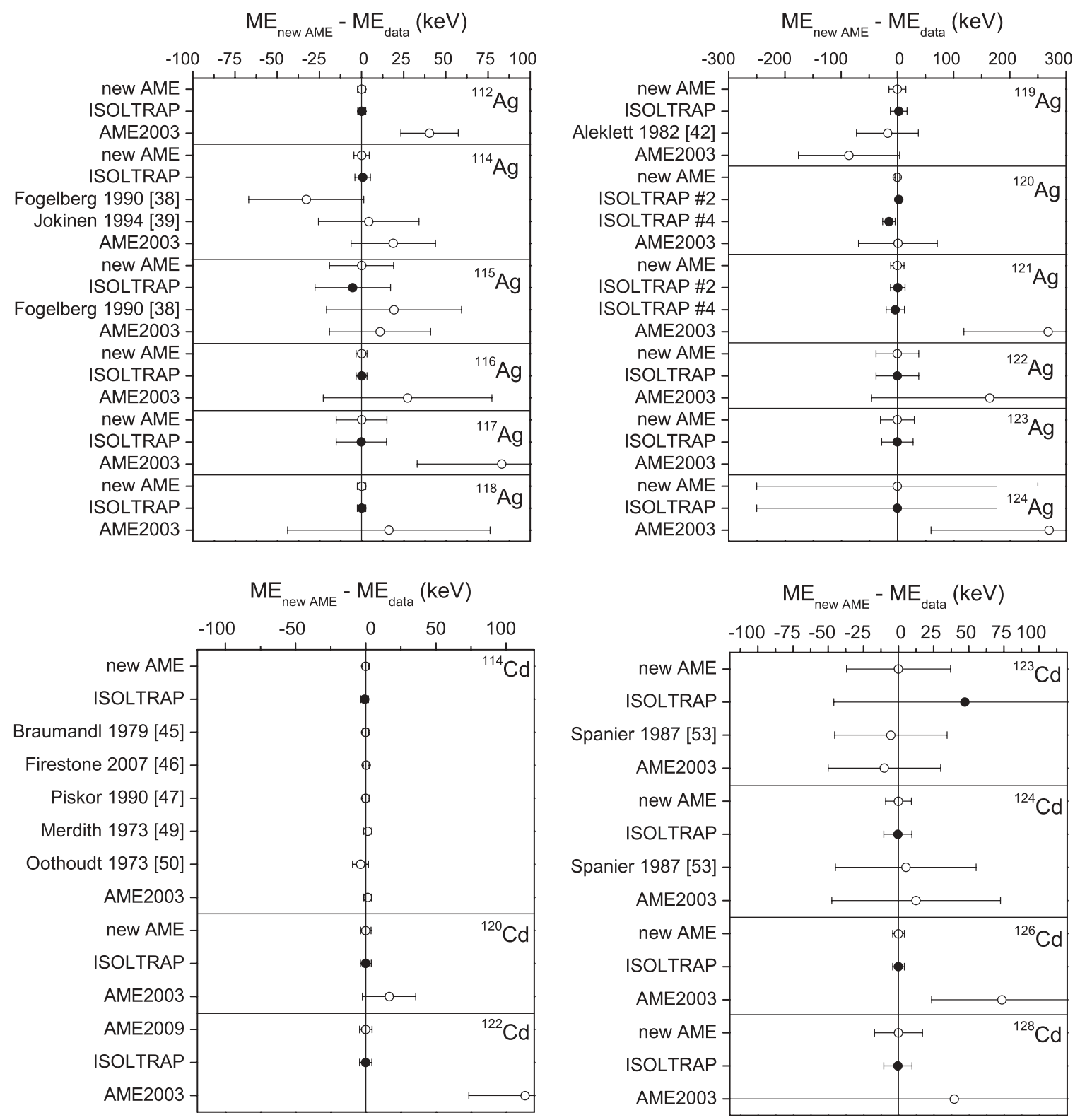

FIG. 3. The mass-excess values $M E$, as determined in the present work (closed circles) and the AME2003 [14] (open symbols) as compared to the new atomic-mass evaluation (zero line, open symbols), which includes our results and all literature values as discussed in the text. Note, that in the cases of ${ }^{115,117,119,121-124} \mathrm{Ag}$ and ${ }^{123} \mathrm{Cd}$ the values determined by ISOLTRAP are treated for an unknown mixture of the isomeric state as described in Ref. [32]. The resonance of ${ }^{118} \mathrm{Ag}$ was assigned to the isomeric state, and therefore the corresponding value for the ground state is plotted. For ${ }^{120,121} \mathrm{Ag}$, where two ISOLTRAP results are contributing to the new AME, the corresponding experiments are indicated.

using the $\beta^{-}$-decay results ( $Q$ value of 7122(100) [42] and 7155 (76) $\mathrm{keV}$ [43]). After the present evaluation, the mass of ${ }^{118} \mathrm{Ag}$ is entirely determined by ISOLTRAP.

$$
{ }^{119} \mathrm{Ag}
$$

The isomeric state ${ }^{119} \mathrm{Ag}^{m}$ is predicted to have an excitation energy of 20(20) keV. For this reason the mass-excess value of ${ }^{119} \mathrm{Ag}$ was corrected to $-78648(14) \mathrm{keV}$. This value agrees with the AME2003 value from the $\beta^{-}$decay of ${ }^{119} \mathrm{Ag}$ with a $Q$ value of 5350(40) $\mathrm{keV}$ [42]. In the new evaluation the ISOLTRAP mass-excess value contributes $97.3 \%$ and the other $2.7 \%$ comes from ${ }^{119} \mathrm{Ag}\left(\beta^{-}\right){ }^{119} \mathrm{Cd}$.

\section{${ }^{120} \mathrm{Ag}$}

The two mass-excess values determined at ISOLTRAP agree within their uncertainties with the AME2003 value, which originates from two measurements of the $\beta^{-}$decay of ${ }^{120} \mathrm{Ag}\left(\beta^{-}\right){ }^{120} \mathrm{Cd}$ with results $Q=8200$ (100) [42] and $Q=8450$ (100) $\mathrm{keV}$ [43]. The value of the new AME is determined $100 \%$ by the weighted average of the two ISOLTRAP measurements.

\section{${ }^{121} \mathrm{Ag}$}

The ISOLTRAP values and the one from AME2003 deviate by about $1.8 \sigma$. The latter was determined by the $\beta^{-}$decay of 
${ }^{121} \mathrm{Ag}$ with a $Q$ value of $6400(120) \mathrm{keV}$ [42]. In the new evaluation the mass-excess value of ${ }^{121} \mathrm{Ag}$ is the weighted average of the two ISOLTRAP results and it is corrected for a potentially present excited state isomere.

$$
{ }^{122} \mathrm{Ag}
$$

The isomeric state ${ }^{122} \mathrm{Ag}^{m}$ has a predicted excitation energy of $80(50) \mathrm{keV}$. Resulting from the isomeric correction the corrected mass-excess value of ${ }^{122} \mathrm{Ag}$ is $-71106(38) \mathrm{keV}$. This ISOLTRAP value agrees with the one extrapolated in AME2003 within its uncertainty. In addition, it also agrees with a recent isochronous mass spectrometry (IMS) measurement at the ESR of GSI [44] but is much more precise. Thus, in the new evaluation the mass-excess value of ${ }^{122} \mathrm{Ag}$ is completely determined by the ISOLTRAP result.

$$
{ }^{123} \mathrm{Ag}
$$

The mass of ${ }^{123} \mathrm{Ag}$ was measured in two experiments (see Table III). Both mass-excess values agree within their uncertainties, but only the latest one is taken into account due to its much higher precision. After applying the isomeric correction mentioned previously, one obtains a mass-excess value of -69548 (30) $\mathrm{keV}$ for ${ }^{123} \mathrm{Ag}$. This mass-excess value differs by about $1.9 \sigma$ from the extrapolated value from AME2003 and by $1.4 \sigma$ from an IMS measurement [-69377(121) keV [44]]. The value of the new AME is entirely determined by the new ISOLTRAP result.

$$
{ }^{124} \mathrm{Ag}
$$

The mass of ${ }^{124} \mathrm{Ag}$ was measured for the first time. The result and the extrapolated value from AME2003 agree within the given uncertainties. The value of the new AME includes a correction for a potentially present isomeric state and it is entirely determined by the ISOLTRAP result.

\section{B. The Cd isotopes}

$$
{ }^{114} \mathrm{Cd}
$$

The measurement performed at ISOLTRAP agrees very well with the value of the AME2003. The mass-excess value of ${ }^{114} \mathrm{Cd}$ was previously determined with a weighting of $70.6 \%$ by the $Q$ values of two measurements of the (n, $\gamma$ ) reaction ${ }^{113} \mathrm{Cd}(\mathrm{n}, \gamma){ }^{114} \mathrm{Cd}(1414(30)$ [45] and $1452(25) \mathrm{keV}$ [46]). A contribution of $10.6 \%$ came from the reaction $Q$ value of ${ }^{114} \mathrm{Cd}(\mathrm{d}, \mathrm{p}){ }^{115} \mathrm{Cd}$ with $Q=3916.30(0.59) \mathrm{keV}$ [47] and $8.2 \%$ by the mass difference between ${ }^{12} \mathrm{C}_{8}{ }^{1} \mathrm{H}_{18}$ and ${ }^{114} \mathrm{Cd}$ given with $237487.6(2.9) \mu \mathrm{u}$ [48]. The mass doublet of ${ }^{116} \mathrm{Cd}^{35} \mathrm{Cl}_{-}{ }^{114} \mathrm{Cd}^{37} \mathrm{Cl}=4348.7(1.2) \mu \mathrm{u}$ [49] ${ }^{114} \mathrm{Cd}$ and the $(\mathrm{p}, \mathrm{t})$ reaction of ${ }^{116} \mathrm{Cd}(Q=-6363(5) \mathrm{keV}$ [50]) influenced the mass excess with $8.1 \%$ and $2.5 \%$, respectively. The ${ }^{114} \mathrm{Cd}$ mass-excess value of the new evaluation is determined $57.9 \%$ by the $(n, \gamma)$ reaction, $28.5 \%$ by the ISOLTRAP result, $6.9 \%$ by the $(\mathrm{d}, \mathrm{p})$ reaction, $5.1 \%$ by the $\mathrm{Cd}-\mathrm{Cl}$ mass doublet, and $1.6 \%$ by the $(\mathrm{p}, \mathrm{t})$ reaction.

$$
{ }^{120} \mathrm{Cd}
$$

The mass-excess value of the AME2003 and the result from ISOLTRAP agree within their uncertainties. Previously the mass-excess value was determined by the ${ }^{124} \mathrm{Sn}\left(\mathrm{d},{ }^{8} \mathrm{Li}\right){ }^{120} \mathrm{Cd}$ reaction and by the ${ }^{124} \mathrm{Sn}\left({ }^{3} \mathrm{He},{ }^{7} \mathrm{Be}\right){ }^{120} \mathrm{Cd}$ reaction with $Q$ values of $-5216(24)$ and $-5098(30) \mathrm{keV}$ [51], respectively. In the new evaluation the mass is entirely determined by the value reported in this work.

\section{${ }^{122} \mathrm{Cd}$}

The ISOLTRAP result deviates by $2.8 \sigma$ from the value of the AME2003, which was determined by a two-proton exchange reaction ${ }^{124} \mathrm{Sn}\left({ }^{18} \mathrm{O},{ }^{20} \mathrm{Ne}\right){ }^{122} \mathrm{Cd}$ with a $Q$ value of $-1246(43) \mathrm{keV}$ [52]. The value of the new AME is completely determined by the result of the present work due to its low uncertainty.

\section{${ }^{123} \mathrm{Cd}$}

This nuclide has a long-lived [1.82(3) s] excited state with an excitation energy of $316.52(23) \mathrm{keV}$. The ISOLTRAP result cannot be assigned to either the ground or isomeric states, so the mass-excess value was corrected to $-77367(93) \mathrm{keV}$. Due to the large uncertainty this corrected value only contributes $16.2 \%$ to the mass value of ${ }^{123} \mathrm{Cd}$, while the main influence comes from the $\beta^{-}$decay of ${ }^{123} \mathrm{Cd} Q$ value $(6115(33) \mathrm{keV}$ [53]). This decay determined the mass of ${ }^{123} \mathrm{Cd} 100 \%$ in the AME2003.

\section{${ }^{124} \mathrm{Cd}$}

The mass-excess value of ${ }^{124} \mathrm{Cd}$ of the AME2003 was determined by its $\beta^{-}$-decay $Q$ value of 4166(39) $\mathrm{keV}$ [53]. This value and the result obtained at ISOLTRAP agree very well with each other. In the new evaluation the mass-excess value is determined $97.8 \%$ by ISOLTRAP and $2.2 \%$ by the $\beta^{-}$-decay $Q$ value.

\section{${ }^{126} \mathrm{Cd}$}

The mass-excess value in AME2003 came from a $\beta^{-}$decay of ${ }^{126} \mathrm{Cd}$ with $Q=5486(36) \mathrm{keV}$ [53]. This result and the weighted average of the two ISOLTRAP values deviate by about $1.5 \sigma$. In the new $\mathrm{AME}$ the ${ }^{126} \mathrm{Cd}$ mass-excess value is determined entirely by the two ISOLTRAP results.

\section{${ }^{128} \mathrm{Cd}$}

The ISOLTRAP measurement agrees very well with the AME2003 value originating from the reaction ${ }^{128} \mathrm{Cd}\left(\beta^{-}\right){ }^{128} \mathrm{In}$, $Q=7070$ (290) $\mathrm{keV}$ [53]. The new value is entirely determined by the present result.

\section{DISCUSSION}

The two-neutron separation energy is given by

$S_{2 n}(N, Z)=M E(N-2, Z)-M E(N, Z)+2 M E(n)$,

where $M E(n)$ is the mass-excess value of a neutron. This energy difference is an indicator for structural changes [10,54]. In Fig. 4 the $S_{2 n}$ values for $Z=47-50$ are compared for odd (top) and even (bottom) $N$. The new mass-excess values as determined at ISOLTRAP lead to a smoothing of the $S_{2 n}$ behavior. Thus the region in the nuclide chart below $Z=50$ does not reveal significant irregularities. 


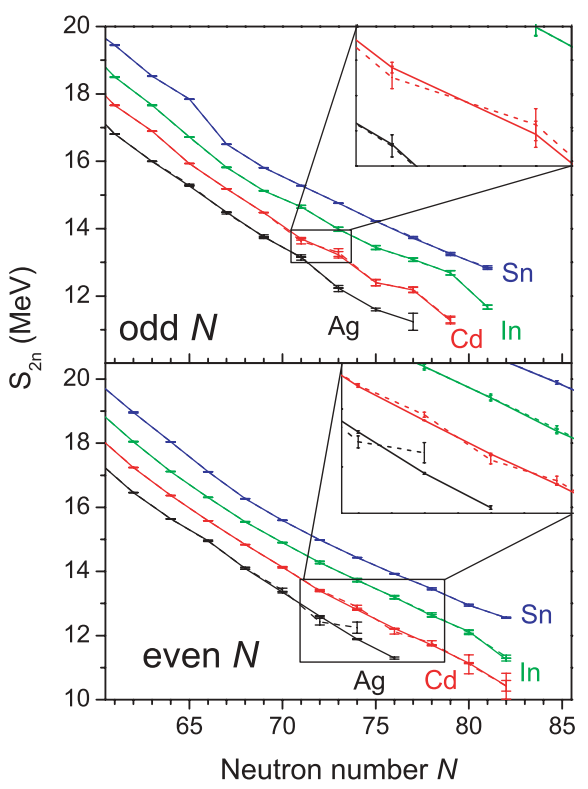

FIG. 4. (Color online) Two-neutron separation energy $S_{2 n}$ as a function of neutron number $N$ for odd (top) and even $N$ (bottom). The insets show the smoothing of the trends for $\mathrm{Cd}$ nuclides with the present results (solid lines) as compared to those extracted from the AME2003 [14] (dashed lines).

A similar tendency of regularity for the neutron-rich nuclei as obtained from the $S_{2 n}$ values can be observed in the GarveyKelson mass relations $[55,56]$ for nuclei with $N>Z$

$$
\begin{aligned}
0= & M E(Z \mp 1, N+1)-M E(Z \pm 1, N-1) \\
& +M E(Z, N-1)-M E(Z, N+1) \\
& +M E(Z \pm 1, N)-M E(Z \mp 1, N) .
\end{aligned}
$$

In these relations between masses of six close-by nuclides various interactions between nucleons as a function of neutron, proton, and mass number (or isospin) such as $\mathrm{p}-\mathrm{p}, \mathrm{n}-\mathrm{n}$, and $\mathrm{p}-\mathrm{n}$ interactions, cancel out to first order. Thus with the knowledge of five of the masses a prediction of the sixth is possible. In principle, there are, in total, 12 different ways to obtain the mass of a nuclide from the Garvey-Kelson mass relations between the mass values of neighboring nuclides. In practice, however, this applies only if all corresponding mass data are actually available (i.e., only in the vicinity of the valley of stability).

Recently, a survey across the entire chart of nuclides was performed [57] using the AME2003 [14]. A root mean square deviation of $\sigma_{\mathrm{rms}} \approx 76 \mathrm{keV}$ of the Garvey-Kelson relations from the actual mass value was obtained for the mass region $A \geqslant 60$, in those cases where all 12 relations were available. If fewer relations were used, the uncertainties were larger. We note that the uncertainties in the Garvey-Kelson relations are not uniquely defined. First, the prediction of masses for even-odd nuclides is more uncertain than for even-even ones and second, the agreement of the Garvey-Kelson relations is better toward closed shells rather than in the average over the entire nuclide chart [57].

The values presented in Fig. 5 are absolute values of the difference between the tabulated masses for $\mathrm{Cd}$ from the $\mathrm{AME}$

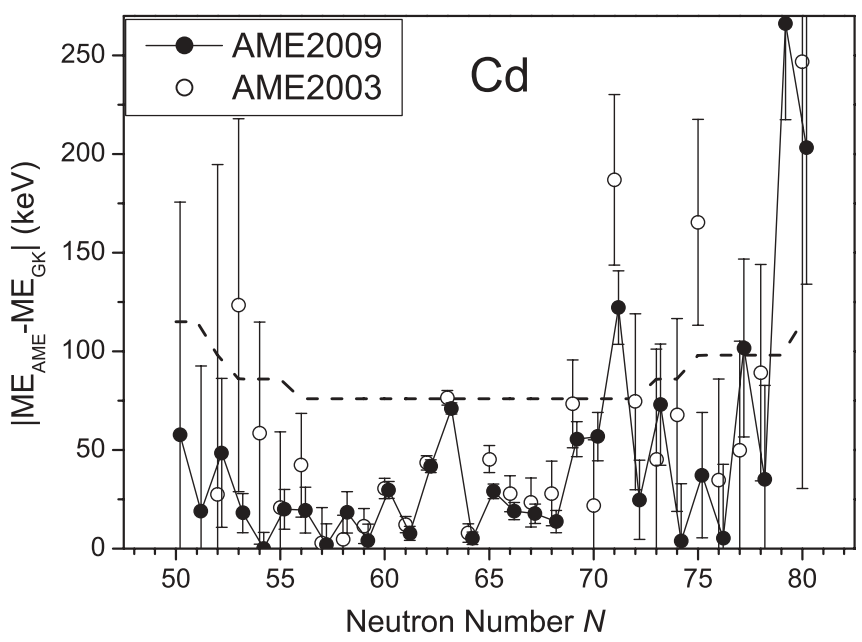

FIG. 5. The absolute value of the differences of mass excesses from either the AME2003 (open circles) or new AME (closed circles) and those calculated with the respective Garvey-Kelson relations. The dashed lines represent the root mean square error $\sigma_{\text {rms }}$ of the Garvey-Kelson relations for the mass region $A \geqslant 60$ as determined in Ref. [57], which varies slightly depending on the number of available relations.

(2003 and new) and the mean of all possible Garvey-Kelson relations using the available masses. The values obtained using the AME2003 (open circles) are compared with the values from the new AME (full circles), which includes also the Pd values from Hager et al. [58]. In general, the new differences are smaller than the old ones, which corresponds to a better agreement between the values obtained from the Garvey-Kelson relations with the experimental data. Note that $\mathrm{Cd}$, with $Z=48$, is close to the proton shell of $\mathrm{Sn}$, which explains the good agreement for most of the values. In contradiction to the idea that the Garvey-Kelson relations work better near closed shells, here we find that the differences between the AME results and the Garvey-Kelson are, on average, larger in the vicinity of a closed shell $(N=50$ or 82 ) than in the midshell region. The most improved value corresponds to the remeasured ${ }^{123} \mathrm{Cd}$. This is consistent with the greater smoothness of the two-neutron separation energy $S_{2 n}$ for even- $N$ Cd nuclides, whereas the the $S_{2 n}$ curve for the odd- $N$ Cd nuclides still deviates from a straight line.

A sensitive probe of structural changes is $\delta V_{p n}$, a double difference of binding energies giving the average interaction of the last proton(s) with the last neutron(s) [11]

$$
\begin{aligned}
\delta V_{p n}(Z, N)= & -\frac{1}{4}(\{M E(Z, N)-M E(Z, N-2)\} \\
& -\{M E(Z-2, N)-M E(Z-2, N-2)\}) .
\end{aligned}
$$

The new ${ }^{114,120,122,124,126,128} \mathrm{Cd}$ mass measurements provide information on $\delta V_{p n}$ below the neutron and proton shell closures $N=82$ and $Z=50$. The values of $\delta V_{p n}$ for eveneven $\mathrm{Sn}$ and $\mathrm{Cd}$ nuclides are plotted in Fig. 6.

When extracted from the mass data of the AME2003 the $\delta V_{p n}(\mathrm{Sn})$ values reveal a zig-zag trend as the number of the valence neutrons decreases toward the closed shell at $N=82$. 


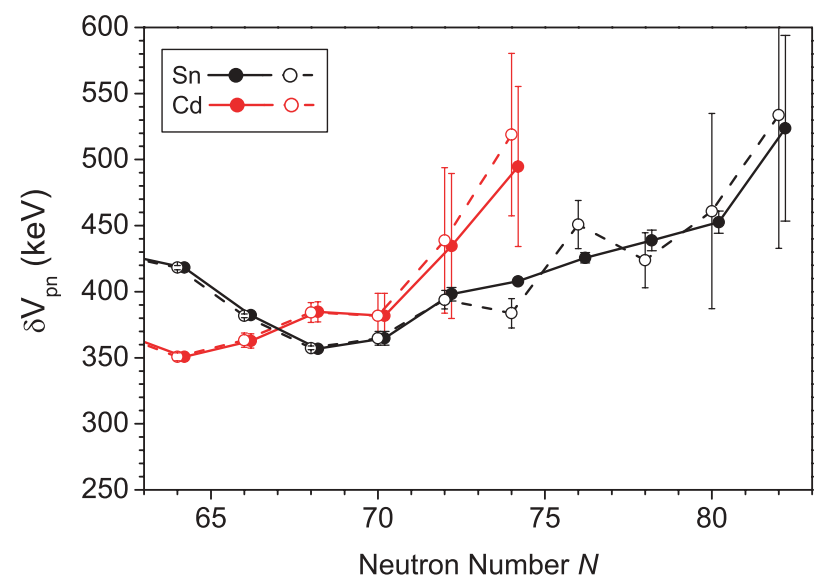

FIG. 6. (Color online) $\delta V_{p n}$ values as a function of neutron number $N$ for the neutron-rich even-even nuclides $\mathrm{Cd}$ and $\mathrm{Sn}$. The full symbols connected with a full line represent $\delta V_{p n}$ values calculated from the present work including values from the AME2003 [14], where no new ISOLTRAP data are available, while the open symbols and dashed lines represent values calculated only from mass values tabled in the AME2003.

The new $\delta V_{p n}(\mathrm{Sn})$ values (solid circles) show a much smoother trend.

In Fig. 7 (left), we compare $\delta V_{p n}$ values as extracted from the new AME for nuclei in the $\mathrm{Sn}$ region near $N=82$ (top) with those in the $\mathrm{Pb}$ region near $N=126$ (bottom). The normal parity orbits in a major shell begin with high $j$ angular momenta and low $n$ principal quantum numbers whereas, near the end of the shell one finds orbits with low $j$ and high $n$. As a result, if both protons and neutrons fill just below or just above a shell at the same time, the overlap between the proton and neutron wave functions are expected to be large. The large $\delta V_{p n}(\mathrm{Sn})$ result for the even-even nuclei, especially at $N=82$, can be explained with this approach.

As seen in Fig. 7 (left), the behavior of the Sn region is very similar to the $\mathrm{Pb}$ region including the sharp increase of Te (similar to Po) after the neutron closed shell, reflecting the larger overlap of neutrons at the beginning of the new shell with the valence protons at the beginning of their new shell. Clearly, this points to the need to measure $\delta V_{p n}\left({ }^{134} \mathrm{Sn}\right)$. This requires a better measurement of the mass of ${ }^{130} \mathrm{Cd}$ and a new measurement for ${ }^{132} \mathrm{Cd}$.

Recently, microscopic density functional theory (DFT) calculations were carried out throughout the nuclear chart. One set of such calculations used an interaction called SKPDMIX which is a Skyme force with mixed volume and surface pairing. The binding energies from these calculations can be used with Eq. (3) to obtain a prediction for $\delta V_{p n}$ values [59]. Even though the DFT SKPDMIX masses are no more accurate on average than about $1 \mathrm{MeV}$, the specific p-n correlations that they include can be isolated and sensitively probed with $\delta V_{p n}$.

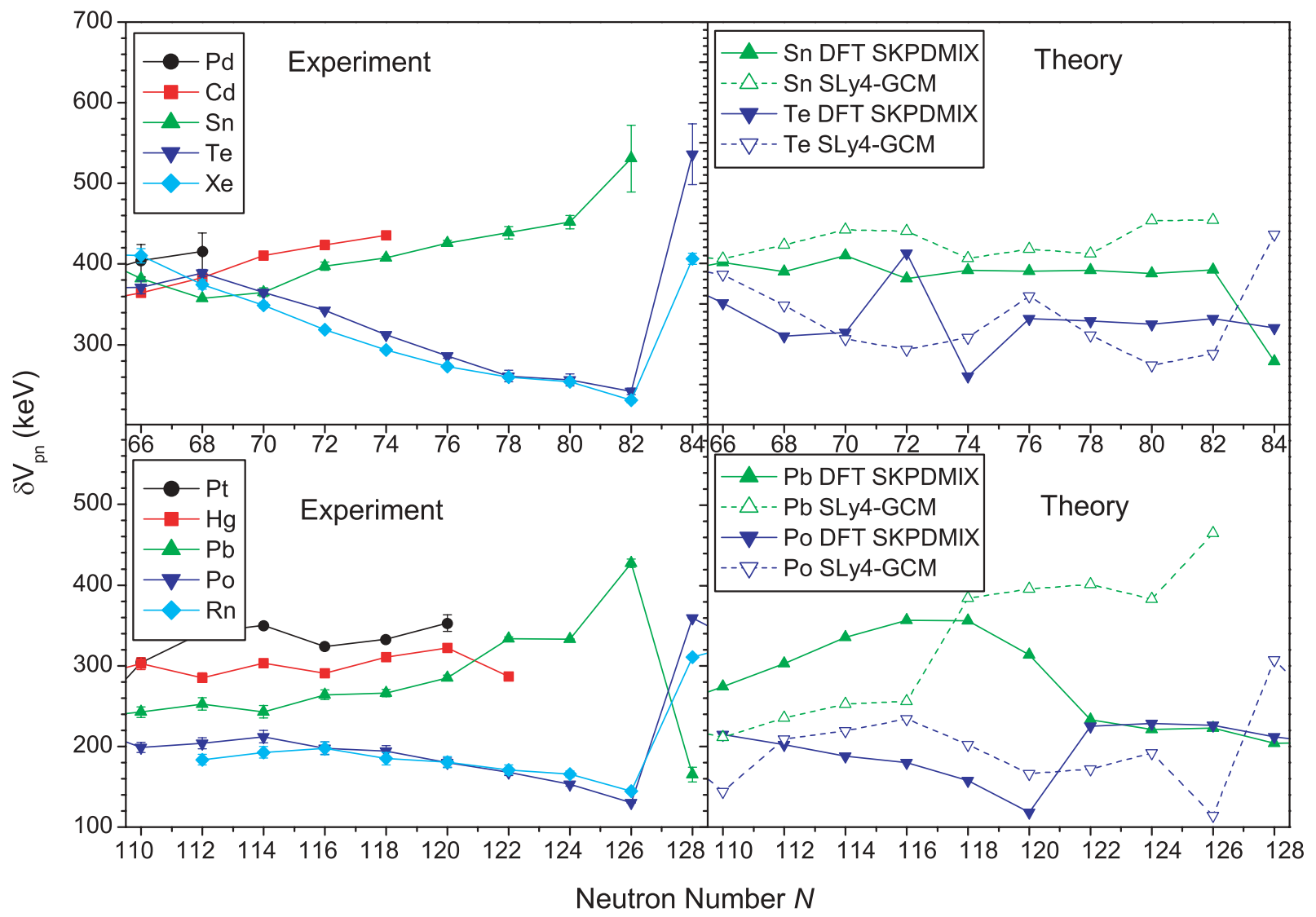

FIG. 7. (Color online) Left: $\delta V_{p n}$ extracted from the new AME as a function of neutron number $N$ for the even- $Z$ nuclides in the vicinity of the proton shell closures $Z=50\left(\mathrm{Pd}, \mathrm{Cd}\right.$, Sn, Te, and $\mathrm{Xe}$ : top) and $Z=82$ (Pt, $\mathrm{Hg}, \mathrm{Pb}, \mathrm{Po}$, and Rn: bottom). Right: $\delta V_{p n}$ extracted from DFT SKPDMIX [59] (full symbols, solid lines) and from SLy4-GCM [60] (open symbols, dashed lines) for Sn and Te (top) and Pb and Po (bottom). 
They match the measured $\delta V_{p n}$ values over most of the region of medium mass and heavy nuclei to, typically, better than $50 \mathrm{keV}$. The most glaring exception to this is near closed shells [see the Pb region in Fig. 7 (bottom)] where it is known that the DFT SKPDMIX does not work very well and is in need of improvement. We now see that, in the Sn region as well [Fig. 7 (top)], the DFT SKPDMIX also faces difficulties near the closed shell while performing better further away. In Fig. 7, we also include results for $\delta V_{p n}$ using an alternate interaction called SLy4 [60] obtained with the SLy4-GCM model. In the second half of the neutron shell, these calculations are somewhat better than the DFT SKPDMIX.

Thus it appears that mass measurements, combined in the form of $\delta V_{p n}$ values, might be useful in testing the p-n correlations embodied in different interactions used in microscopic calculations, and perhaps, thereby, foster improvements in such interactions. The other application of $\delta V_{p n}$ values discussed previously, namely the behavior near shell closures, shows that these proton-neutron interactions can be interpreted directly, close to doubly magic nuclei, in terms of the overlaps of the last proton and neutron wave functions. The characteristic bifurcating behavior, now seen in both the $\mathrm{Sn}$ and $\mathrm{Pb}$ regions, may be useful in the study of shell structure as a harbinger of double magicity in new regions far from stability where masses may be the only obtainable observables.

\section{SUMMARY}

Precision mass measurements of ten neutron-rich $\mathrm{Ag}$ and seven neutron-rich $\mathrm{Cd}$ nuclides are reported with an improvement of a factor up to 30 in experimental uncertainties such as in the case of ${ }^{121} \mathrm{Ag}$. Most of the mass values were determined directly for the first time. The value of ${ }^{124} \mathrm{Ag}$ was measured for the first time. Measurements in this mass region suffer from the presence of long-lived excited isomeric states as discussed for ${ }^{115,117,119,121-124} \mathrm{Ag}$ and ${ }^{123} \mathrm{Cd}$. For those cases the experimental results were corrected as described in the AME2003 [14]. All corrected mass values were included in a mass network update. The resulting masses are discussed with respect to their values in the AME2003.
The new mass values lead to corresponding corrections of the two-neutron separation energies $S_{2 n}$. Kinks in the $S_{2 n}$-versus- $N$ plots found for the previous values disappeared, and in general, a smooth behavior is observed. The GarveyKelson relations show an improved consistency within the uncertainties. Similarly, the $\delta V_{p n}$ values were revised and show smoother behavior in the $\mathrm{Cd}-\mathrm{Sn}$ region. These values are now available for almost the full range of $\mathrm{Sn}$ nuclides between the magic neutron numbers 50 and 82 . In general, a smooth behavior in the region of the mass surface below $Z=50$ toward the neutron-shell closure $N=82$ is observed. The behavior of $\delta V_{p n}$ across the $N=82$ shell closure very closely resembles what was seen in the $\mathrm{Pb}$ region prior to the recent determination of $\delta V_{p n}$ for ${ }^{210} \mathrm{~Pb}$ [16]. This emphasizes the need for a new measurement of ${ }^{132} \mathrm{Cd}$ (and an improved value for the mass of ${ }^{130} \mathrm{Cd}$ ), to give $\delta V_{p n}$ for ${ }^{134} \mathrm{Sn}$ and conclusively test whether these two doubly magic regions behave the same throughout.

Similarly, for investigations of the $S_{2 n}$ values, GarveyKelson relations, and $\delta V_{p n}$ values for odd- $N \mathrm{Cd}$ nuclides, a remeasurement of the ${ }^{119-125} \mathrm{Cd}$ masses is required. This is particularly difficult due to the presence of isomerism as described previously. Finally, the new mass values allowed further tests of the $\mathrm{p}$ - $\mathrm{n}$ correlations in calculations using the DFT SKDMIX and the SLy4-GCM showing that the DFT SKPDMIX does not reproduce the data well near the closed shells, as expected, but that the SLy4-GCM calculations perform noticeably better in these regions.

\section{ACKNOWLEDGMENTS}

This work was supported by the German Federal Ministry for Education and Research (BMBF) (06GF186I, 06MZ215), German DFG under Grant No. Ko. 142/112-1, Helmholtz Association for National Research Centers (VH-NG-037), US Department of Energy (DE-FG02-91ER-40609), the French IN2P3, Turkish Atomic Energy Authority (TAEK) 04K120100-4, the EU FP6 Program (MEIF-CT-2006-042114 and EURONS DS Project/515768 RIDS), and the MaxPlanck Society. R.B.C. thanks for funding by the Humboldt foundation. We are grateful to the members of the ISOLDE technical group for their support.
[1] M. P. Bradley, J. V. Porto, S. Rainville, J. K. Thompson, and D. E. Pritchard, Phys. Rev. Lett. 83, 4510 (1999).

[2] S. Rainville, J. K. Thompson, D. E. Pritchard, Science 303, 334 (2004).

[3] M. Redshaw, E. Wingfield, J. McDaniel, and E. G. Myers, Phys. Rev. Lett. 98, 053003 (2007).

[4] M. Mukherjee et al., Phys. Rev. Lett. 93, 150801 (2004).

[5] M. Smith et al., Phys. Rev. Lett. 101, 202501 (2008).

[6] R. Ringle et al., Phys. Rev. C 75, 055503 (2007).

[7] K. Blaum, Phys. Rep. 425, 1 (2006).

[8] L. Schweikhard and G. Bollen (Eds.), Int. J. Mass Spectrom. 251, (2/3) (2006).

[9] M. Mukherjee et al., Eur. Phys. J. A 35, 1 (2008).
[10] M. Dworschak et al., Phys. Rev. Lett. 100, 072501 (2008).

[11] J.-Y. Zhang, R. F. Casten, and D. S. Brenner, Phys. Lett. B 227, 1 (1989).

[12] R. B. Cakirli, D. S. Brenner, R. F. Casten, and E. A. Millman, Phys. Rev. Lett. 94, 092501 (2005).

[13] D. S. Brenner, R. B. Cakirli, and R. F. Casten, Phys. Rev. C 73, 034315 (2006).

[14] G. Audi, A. H. Wapstra, and C. Thibault, Nucl. Phys. A 729, 337 (2003).

[15] D. Neidherr et al., Phys. Rev. Lett. 102, 112501 (2009).

[16] L. Chen et al., Phys. Rev. Lett. 102, 139904(E) (2009).

[17] B. Franzke, H. Geissel, and G. Münzenberg, Mass Spectrom. Rev. 27, 428 (2008).

[18] E. Kugler, Hyperfine Interact. 129, 23 (2000). 
[19] Y. Jading et al., Nucl. Instrum. Methods Phys. Res., Sect. B 126, 76 (1997).

[20] U. Köster et al., Nucl. Instrum. Methods Phys. Res., Sect. B 204, 347 (2003).

[21] E. Bouquerel, R. Catherall, M. Eller, J. Lettry, S. Marzari, and T. Stora, Nucl. Instrum. Methods Phys. Res., Sect. B 266, 4298 (2008).

[22] F. Herfurth et al., Nucl. Instrum. Methods Phys. Res., Sect. A 469, 254 (2001).

[23] F. Herfurth, Nucl. Instrum. Methods Phys. Res., Sect. B 204, 587 (2003).

[24] H. Raimbault-Hartmann, D. Beck, G. Bollen, M. König, H.-J. Kluge, E. Schark, J. Stein, S. Schwarz, and J. Szerypo, Nucl. Instrum. Methods Phys. Res., Sec. B 126, 378 (1997).

[25] G. Savard, S. Becker, G. Bollen, H.-J. Kluge, R. Moore, T. Otto, L. Schweikhard, H. Stolzenberg, and U. Wiess, Phys. Lett. A 158, 247 (1991).

[26] G. Gräff, H. Kalinowsky, and J. Traut, Z. Phys. A 297, 35 (1980).

[27] C. Yazidjian, K. Blaum, R. Ferrer, F. Herfurth, A. Herlert, and L. Schweikhard, Hyperfine Interact. 173, 181 (2006).

[28] M. König, G. Bollen, H.-J. Kluge, T. Otto, and J. Szerypo, Int. J. Mass Spectrom. 142, 95 (1995).

[29] A. Kellerbauer, K. Blaum, G. Bollen, F. Herfurth, H.-J. Kluge, M. Kuckein, E. Sauvan, C. Scheidenberger, and L. Schweikhard, Eur. Phys. J. D 22, 53 (2003).

[30] M. Mukherjee et al., Eur. Phys. J. A 35, 31 (2008).

[31] R. Catherall, J. Lettry, S. Gilardoni, and U. Köster, Nucl. Instrum. Methods Phys. Res., Sect. B 204, 235 (2003).

[32] A. H. Wapstra, G. Audi, and C. Thibault, Nucl. Phys. A 729, 129 (2003).

[33] D. Beck et al., Eur. Phys. J. A 8, 307 (2000).

[34] M. Breitenfeldt et al., Phys. Rev. C 80, 035805 (2009).

[35] G. Audi, W. G. Davies, and G. E. Lee-Whiting, Nucl. Instrum. Methods Phys. Res., Sect. A 249, 443 (1986).

[36] H. Inoue, J.-Z. Ruan, S. Yasukawa, and Y. Yoshizawa, Nucl. Phys. 38, 50 (1962).

[37] R. H. Nussbaum, A. H. Wapstra, M. J. Sterk, and R. E. W. Kropveld, Physica 21, 77 (1954).

[38] B. Fogelberg, Y. Zongyuan, B. Ekström, E. Lund, K. Aleklett, and L. Sihver, Z. Phys. A 337, 251 (1990).
[39] A. Jokinen, Ph.D. thesis, University of Jyväskylä, 1994.

[40] E. L. Bahn, B. D. Pate, R. D. Fink, and C. D. Coryell, Phys. Rev. 136, B603 (1964).

[41] Z. Matumoto and T. Tamura, J. Phys. Soc. Jpn. 44, 1070 (1978).

[42] K. Aleklett, P. Hoff, E. Lund, and G. Rudstam, Phys. Rev. C 26, 1157 (1982).

[43] A. Aprahamian, D. S. Brenner, R. Gill, A. Piotrowski, and R. F. Casten (private communication).

[44] B. Sun et al., Nucl. Phys. A 812, 1 (2008).

[45] F. Braumandl, K. Schreckenbach, and T. Von Egidy, Nucl. Instrum. Methods 166, 243 (1979).

[46] R. B. Firestone, H. D. Choi, R. M. Lindstrom, G. L. Molnar, S. F. Mughabghab, R. Paviotti-Corcuera, Zs. Revay, V. Zerkin, and C. M. Zhou, IAEA STI/PUB/1263, 251 pp (2007).

[47] S. Piskoř and W. Schäferlingová, Nucl. Phys. A 510, 301 (1990).

[48] R. A. Damerow, R. R. Ries, and W. H. Johnson, Phys. Rev. 132, 1673 (1963).

[49] J. O. Meredith, F. C. G. Southon, R. C. Barber, P. Williams, and H. E. Duckworth, Int. J. Mass Spectrom. Ion Phys. 10, 359 (1973).

[50] M. A. Oothoudt and N. M. Hintz, Nucl. Phys. A 213, 221 (1973).

[51] W. F. Steele, P. A. Smith, J. E. Finck, and G. M. Crawley, Nucl. Phys. A 266, 424 (1976).

[52] J. Guo, K. Zhao, X. Lu, Y. Cheng, T. Li, C. Fu, and S. Li, Chin. J. Nucl. Phys. 19, 180 (1997).

[53] L. Spanier, K. Aleklett, B. Ekström, and B. Fogelberg, Nucl. Phys. A 474, 359 (1987).

[54] C. Guénaut et al., J. Phys. G 31, S1765 (2005).

[55] G. T. Garvey and I. Kelson, Phys. Rev. Lett. 16, 197 (1966).

[56] G. T. Garvey, W. J. Gerace, R. L. Jaffe, I. Talmi, and I. Kelson, Rev. Mod. Phys. 41, S1 (1969).

[57] J. Barea, A. Frank, J. G. Hirsch, P. V. Van Isacker, S. Pittel, and V. Velázquez, Phys. Rev. C 77, 041304(R) (2008).

[58] U. Hager et al., Phys. Rev. C 75, 064302 (2007).

[59] M. Stoitsov, R. B. Cakirli, R. F. Casten, W. Nazarewicz, and W. Satuła, Phys. Rev. Lett. 98, 132502 (2007).

[60] M. Bender, G. F. Bertsch, and P.-H. Heenen, Phys. Rev. C 73, 034322 (2006). 